\title{
Clumpy photon-dominated regions in Carina
}

\section{I. [CI] and mid-J CO lines in two $4^{\prime} \times 4^{\prime}$ fields}

\author{
C. Kramer ${ }^{1}$, M. Cubick ${ }^{1}$, M. Röllig ${ }^{3}$, K. Sun ${ }^{1}$, Y. Yonekura ${ }^{2}$, M. Aravena ${ }^{3}$, F. Bensch ${ }^{3}$, F. Bertoldi ${ }^{3}$, L. Bronfman ${ }^{4}$, \\ M. Fujishita ${ }^{5}$, Y. Fukui ${ }^{5}$, U. U. Graf ${ }^{1}$, M. Hitschfeld ${ }^{1}$, N. Honingh ${ }^{1}$, S. Ito ${ }^{5}$, H. Jakob ${ }^{1}$, K. Jacobs ${ }^{1}$, U. Klein ${ }^{3}$, \\ B.-C. Koo $^{6}$, J. May ${ }^{4}$, M. Miller ${ }^{1}$, Y. Miyamoto ${ }^{5}$, N. Mizuno ${ }^{5}$, T. Onishi ${ }^{5}$, Y.-S. Park ${ }^{6}$, J. L. Pineda ${ }^{3}$, D. Rabanus ${ }^{1}$, \\ H. Sasago ${ }^{5}$, R. Schieder ${ }^{1}$, R. Simon ${ }^{1}$, J. Stutzki ${ }^{1}$, N. Volgenau ${ }^{1}$, and H. Yamamoto ${ }^{5}$
}

1 KOSMA, I. Physikalisches Institut, Universität zu Köln, Zülpicher Straße 77, 50937 Köln, Germany e-mail: kramer@ph1.uni-koeln.de

2 Department of Physical Science, Osaka Prefecture University, Osaka 599-8531, Japan

3 Argelander-Institut für Astronomie, Auf dem Hügel 71, 53121 Bonn, Germany

4 Departamento de Astronomía, Universidad de Chile, Casilla 36-D, Santiago, Chile

5 Department of Astrophysics, Nagoya University, Chikusa-ku, Nagoya 464-8602, Japan

${ }^{6}$ Seoul National University, Seoul 151-742, Korea

Received 8 May 2007 / Accepted 29 October 2007

\section{ABSTRACT}

\begin{abstract}
Context. The Carina region is an excellent astrophysical laboratory for studying the feedback mechanisms of newly born, very massive stars within their natal giant molecular clouds (GMCs) at only $2.35 \mathrm{kpc}$ distance.

Aims. We use a clumpy PDR model to analyse the observed intensities of atomic carbon and CO and to derive the excitation conditions of the gas.

Methods. The NANTEN2-4 m submillimeter telescope was used to map the [C I $]{ }^{3} \mathrm{P}_{1}-{ }^{3} \mathrm{P}_{0},{ }^{3} \mathrm{P}_{2}-{ }^{3} \mathrm{P}_{1}$ and $\mathrm{CO} 4-3$, 7-6 lines in two $4^{\prime} \times 4^{\prime}$ regions of Carina where molecular material interfaces with radiation from the massive star clusters. One region is the northern molecular cloud near the compact OB cluster Tr 14, and the second region is in the molecular cloud south of $\eta$ Car and $\operatorname{Tr} 16$. These data were combined with ${ }^{13} \mathrm{CO}$ SEST spectra, HIRES/IRAS $60 \mu \mathrm{m}$ and $100 \mu \mathrm{m}$ maps of the FIR continuum, and maps of $8 \mu \mathrm{m}$ IRAC/Spitzer and MSX emission.

Results. We used the HIRES far-infrared dust data to create a map of the FUV field heating the gas. The northern region shows an FUV field of a few $10^{3}$ in Draine units while the field of the southern region is about a factor 10 weaker. While the IRAC $8 \mu$ m emission lights up at the edges of the molecular clouds, $\mathrm{CO}$ and also [C I] appear to trace the $\mathrm{H}_{2}$ gas column density. The northern region shows a complex velocity and spatial structure, while the southern region shows an edge-on PDR with a single Gaussian velocity component. We constructed models consisting of an ensemble of small spherically symmetric PDR clumps within the $38^{\prime \prime}$ beam ( $\left.0.43 \mathrm{pc}\right)$, which follow canonical power-law mass and mass-size distributions. We find that an average local clump density of $2 \times 10^{5} \mathrm{~cm}^{-3}$ is needed to reproduce the observed line emission at two selected interface positions.

Conclusions. Stationary, clumpy PDR models reproduce the observed cooling lines of atomic carbon and CO at two positions in the Carina Nebula.
\end{abstract}

Key words. ISM: clouds - ISM: structure - ISM: individual objects: Carina

\section{Introduction}

\subsection{The importance of atomic carbon}

The fine structure lines of atomic carbon are strong coolants of the interstellar medium (ISM) throughout the universe: in Galactic clouds (e.g. Jakob et al. 2007), in the Milky Way as a whole (Fixsen et al. 1999), as well as in external galaxies (Bayet et al. 2006; Kramer et al. 2005) up to high redshifts (Weiss et al. 2003). Large-scale observations of Galactic clouds show [C I] emission coincident with that of CO. This holds for giant molecular clouds (GMCs) (Sakai et al. 2006), as well as for rather quiescent dark clouds (Tatematsu et al. 1999). It has therefore been suggested that $[\mathrm{CI}]$ is a more reliable tracer of the molecular cloud masses in external galaxies than $\mathrm{CO}$ (Papadopoulos et al. 2004). However, all steady-state models of photon-dominated regions (PDRs) predict that atomic carbon is formed in a surface layer by recombination of $\mathrm{C}^{+}$and dissociation of $\mathrm{CO}$. This appears to be in contradiction with atomic carbon emission which is observed even at large distances from UV sources. The dynamics of the ISM may play a decisive role, allowing the distribution of $[\mathrm{CI}]$ to be explained with time dependent chemical models (Oka et al. 2004; Störzer et al. 1997).

However, stationary, but clumpy PDR models have been successful in explaining e.g. extended [C I $]{ }^{3} \mathrm{P}_{1}-{ }^{3} \mathrm{P}_{0}$ and ${ }^{13} \mathrm{CO} 2-1$ emission in S 140 (Spaans 1996; Spaans \& van Dishoeck 1997). Inside a clumpy PDR, the UV radiation can penetrate much deeper than it would be possible in a homogeneous layer (Boissé 1990). Meixner \& Tielens (1993) showed that the line intensities of all lines are enhanced compared to a homogeneous model with the same average density. Juvela et al. (2001) presented a model to apply Monte Carlo radiative transfer calculations to the results of magnetohydrodynamic calculations to account for a clumpy and turbulent structure. They also found that local density condensations lead to an increased cooling efficiency and reduced photon trapping. Another approach is to describe both the turbulent velocity field and the density structure by their 

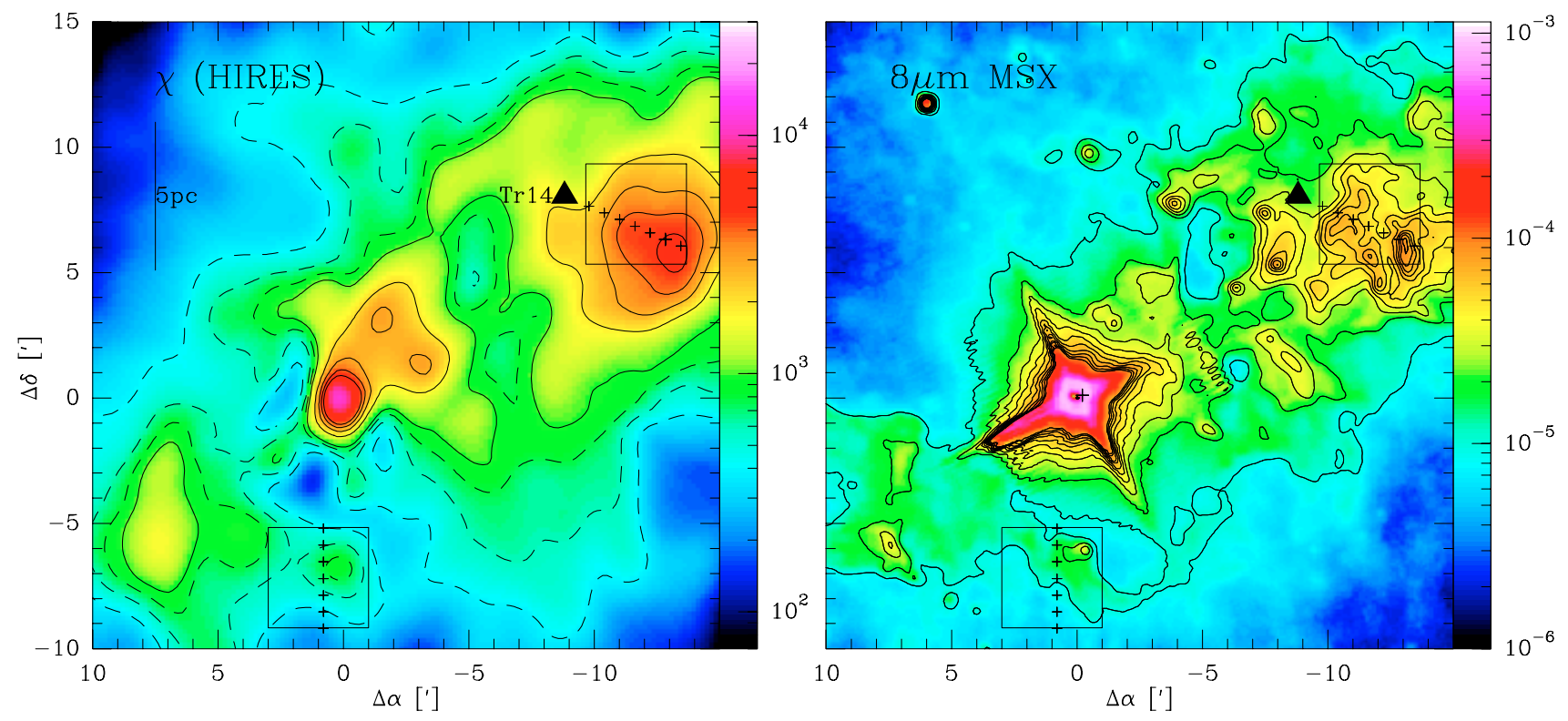

Fig. 1. Overview of the Carina clouds near $\eta$ Car lying at 0/0 10:45:03.59 -59:41:04.3 (J2000) at the center of the Tr 16 cluster. Left: estimate of the FUV flux $\chi$ in Draine units at a resolution of $\sim 1^{\prime}$ and derived from HIRES/IRAS. Dashed contours range from 400 to 800 in steps of 200. Solid contours range from $10^{3}$ to $5 \times 10^{3}$ in steps of $10^{3}$. Right: MSX emission at $8 \mu \mathrm{m}$ at a resolution of $\sim 20^{\prime \prime}$. Contours are 1 to 10 in steps of $1 \times 10^{-5} \mathrm{~W} \mathrm{~m}^{-2} \mathrm{sr}^{-1}$ (cf. Figs. 7, 11 in Rathborne et al. 2002). Note the diffraction artefacts near $\eta$ Car. The center position of the OB cluster Tr 14 is marked by a triangle. The two $4^{\prime} \times 4^{\prime}$ regions mapped with NANTEN2 are delineated by boxes. Crosses in the left panel mark two cuts discussed in the text.

statistical properties. Hegmann et al. (2007) calculate CO cooling rates based on a stochastic radiative transfer model, which accounts for density and velocity fluctuations with a finite correlation length.

Here, we study the distribution of atomic carbon and warm $\mathrm{CO}$ in the Carina star forming region and test clumpy models using the KOSMA- $\tau$ PDR model (Störzer et al. 1996; Röllig et al. 2007) to derive the excitation conditions of the gas. Spaans et al. model the inhomogeneous structure with a Monte-Carlo code, assuming a fixed clump/interclump density ratio of 10 . In contrast, we assume that all emission analyzed stems from the clumps. The influence of the interclump medium or a halo leading to pre-shielding of $\mathrm{CO}$ has been discussed by Bensch (2006) and Bensch et al. (2003), who also used KOSMA- $\tau$, but is not considered here. To describe the clumpy structure, we assume that the clumps are distributed following the canonical clump mass and mass-size distributions which have been found in molecular clouds.

\subsection{The Carina nebula}

The Carina nebula is a very prominent southern star-forming region at a distance of $2.35 \mathrm{kpc}$ (Smith 2006b). This giant molecular cloud was first observed by Grabelsky et al. (1988) as part of the Columbia CO 1-0 survey of the Milky Way. They derived a total mass of $6.7 \times 10^{5} M_{\odot}$ and an effective radius of $66 \mathrm{pc}$. In total 65 O-type stars, including 6 of the 11 O3-type stars known in the Milky Way (Smith 2006a), produce a strong UV field and strong stellar winds which interact vigorously with the material in the surrounding molecular clouds. This high concentration of the earliest-type stars is unique in the Galaxy and may serve as a template for more extreme but more distant regions containing stellar super clusters like the central regions of NGC 253 or the Antennae (Bayet et al. 2006; Schulz et al. 2007). The Carina nebula extends more than 4 square degrees on the sky. It exhibits a peculiar morphological structure on all scales.
Massive stars are being born in several regions within molecular condensations, located mainly to the south-east and north-west of the cluster Trumpler 16, which is centered at $\eta$ Car, a luminous blue variable star. $\eta$ Car is embedded in the Homunculus nebula (e.g. Smith 2006a) and its luminosity has changed drastically and repeatedly in the past centuries, leading to strong variations of the FUV field impinging on the surrounding clouds. Its current luminosity of $5 \times 10^{6} L_{\odot}$ makes it one of the brightest celestial infrared objects (Cox 1995). In the northern part of the Carina nebula, the stellar population is dominated by the cluster Tr 14. Recently, Oberst et al. (2006) reported the detection of the [N II] line at $205 \mu \mathrm{m}$ at the radio peak of the Carina II H II region using SPIFI at AST/RO. They compared their data with [C II] data (Mizutani et al. 2004, 2002) and concluded that about a third of the [C II] emission stems from a low-density ionized medium. For a recent overview of the Carina region, see Smith \& Brooks (2007).

Carina was mapped at $\sim 3^{\prime}$ resolution in the $\mathrm{CO}$ 4-3 and $[\mathrm{CI}]{ }^{3} \mathrm{P}_{1}-{ }^{3} \mathrm{P}_{0}$ lines by Zhang et al. (2001) using the AST/RO $1.7 \mathrm{~m}$ telescope. These AST/RO data show large-scale [C I] emission, coextensive with $\mathrm{CO}$ 4-3.

Here, we have used the $4 \mathrm{~m}$ NANTEN2 telescope to map ${ }^{12} \mathrm{CO}$ in the rotational transitions $J=4-3,7-6$, and $[\mathrm{CI}]$ in the fine structure transitions ${ }^{3} \mathrm{P}_{1}-{ }^{3} \mathrm{P}_{0}$, and ${ }^{3} \mathrm{P}_{2}-{ }^{3} \mathrm{P}_{1}$ (henceforth $1-0$ and 2-1) at sub-arcminute resolutions in two $4^{\prime} \times 4^{\prime}$ regions of Carina (Fig. 1).

One region to the north of $\eta$ Car covers the cloud interface with $\operatorname{Tr} 14$. The second region covers another cloud interface to the south of $\eta$ Car and Tr 16.

The northern molecular cloud to the west of Tr 14 (Fig. 1) contains several sites of massive star formation. Diffuse PAH $3.29 \mu \mathrm{m}$ emission has been found by Rathborne et al. (2002) to trace the sharp edge of the cloud. Peak intensities at the edge towards the Car I H II region are almost 10 times higher than in the other regions of Carina. Car I-E is interacting with the front face of the GMC and creating a PDR seen edge-on 


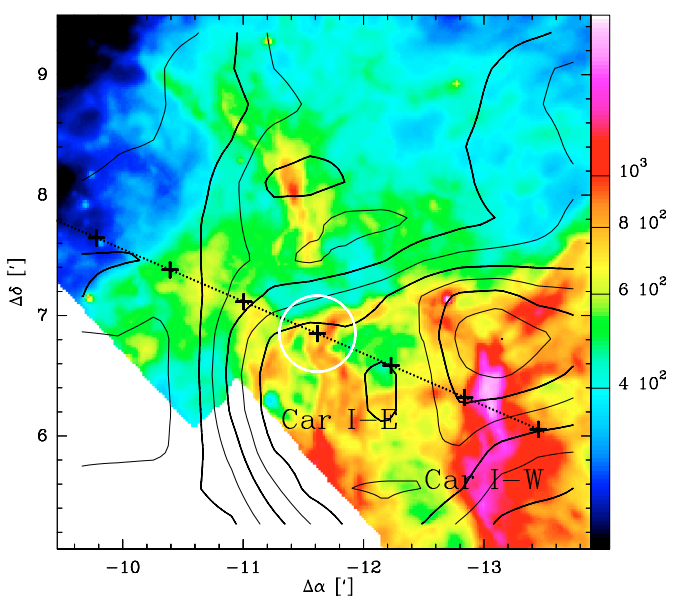

Fig. 2. Northern field: Contours of integrated CO 4-3 intensities (cf. Fig. 3) overlayed on $8 \mu \mathrm{m} \mathrm{IRAC/Spitzer} \mathrm{emission} \mathrm{in} \mathrm{units} \mathrm{of} \mathrm{MJy} / \mathrm{sr}$. Car I-E and Car I-W are two H II regions described e.g. by Brooks et al. (2003) (cf. Fig. 3 for more details).

(Brooks et al. 2003; Brooks et al. 2001). Car I-W manifests a second PDR seen face-on (cf. Fig. 2).

The CO 2-1 map of Cox (1995) shows a sharp rim of gas delineating the southern molecular cloud, located south of $\eta$ Car and the Tr 16 cluster. Several IRAS sources are located along the edge. A prominent peak in PAH $3.29 \mu \mathrm{m}$ emission is the ultra compact H II region IRAS 10430-5931 (Rathborne et al. 2002).

\section{NANTEN2 observations}

We used the new NANTEN2 $4 \mathrm{~m}$ telescope situated at $4865 \mathrm{~m}$ altitude at Pampa La Bola in northern Chile to map two regions in Carina in $\mathrm{CO} 4-3$ and 7-6, and in the two fine structure transitions of atomic carbon. Observations were conducted in 2006 between September 21 and October 6 with a dual-channel $460 / 810 \mathrm{GHz}$ receiver installed for verifying the telescope submillimeter performance. Double-sideband (DSB) receiver temperatures were $\sim 250 \mathrm{~K}$ in the lower channel and $\sim 750 \mathrm{~K}$ in the upper one. The intermediate frequencies (IF) are $4 \mathrm{GHz}$ and $1.5 \mathrm{GHz}$, respectively. The latter IF allows simultaneous observations of the CO 7-6 line in the lower and the [C I] 2-1 line in the upper sideband. These two lines are observed simultaneously with one of the lines in the $460 \mathrm{GHz}$ channel. As backends, we used two acousto-optical spectrometers (AOS) with a bandwidth of $1 \mathrm{GHz}$ and a channel resolution of $0.37 \mathrm{~km} \mathrm{~s}^{-1}$ at $460 \mathrm{GHz}$ and $0.21 \mathrm{~km} \mathrm{~s}^{-1}$ at $806 \mathrm{GHz}$.

The pointing was checked regularly on Jupiter, IRC +10216 , and IRc2 in Orion A. The applied corrections were always smaller than $20^{\prime \prime}$, and usually less than $10^{\prime \prime}$. The atmospheric transmission was derived by measuring the atmospheric emission at the reference position. Carina was observed between $\sim 32^{\circ}$ and $\sim 54^{\circ}$ elevation. Spectra of the two frequency bands were calibrated separately, and sideband imbalances were corrected using the atmospheric model atm (Pardo et al. 2001). We used a reference position at 10.50m.09:3 -59.18.33".7 (J2000), $\sim 45^{\prime}$ to the north-east of $\eta$ Car, which was observed to be free of emission by Yonekura et al. (2005). Observations were conducted on-the-fly (OTF), scanning in right ascension at a speed of $2.5^{\prime \prime} / \mathrm{s}$ and with a sampling interval of $10^{\prime \prime}$. [C I] emission of the northern field was also observed scanning in declination. The reference position was observed at the beginning of each OTF scanning line, i.e. every $1.6 \mathrm{~min}$ in time. To complete a single scan of the $4^{\prime} \times 4^{\prime}$ map area took $\sim 1 \mathrm{~h}$ of total observing time. ON-source integration times per position on a $10^{\prime \prime}$ grid range between $24 \mathrm{~s}$ (for $\mathrm{CO} 4-3 \& 7-6$ in the northern field) and $4 \mathrm{~s}$. The total observing time was $10 \mathrm{~h}$.

The half power beamwidths (HPBWs) and main beam efficiencies $\left(B_{\text {eff }}\right)$ were determined from continuum cross scans on Jupiter (Simon et al. 2007). For the latter, we linearly interpolated the Jovian brightness temperatures listed by Griffin et al. (1986) to the observed frequencies. The HPBWs deconvolved from the observed full widths at half maximum ( $F W H M)$, are $38^{\prime \prime} .0$ and $26{ }^{\prime \prime} 5$, in the lower and upper receiver band, respectively. Beam efficiencies are $50 \%$ and $45 \%$, respectively. The forward efficiency $\left(F_{\text {eff }}\right)$ was derived from skydips and was found to be $86 \%$ for both frequency bands. The raw data taken at the telescope was recalibrated to the $T_{\mathrm{A}}^{*}$ antenna temperature scale and then multiplied by $F_{\text {eff }} / B_{\text {eff }}$ to scale to main beam temperatures. All data presented here are on the $T_{\mathrm{mb}}$ scale. We fitted and subtracted 2 nd order polynomials from all spectra. In a few cases, we subtracted higher-order polynomials.

During the test campaign in the second half of 2006, we were able to roughly measure the error beam amplitudes of the NANTEN2 antenna (Simon et al. 2007). Continuum measurements of the solar and lunar edges allowed to measure the extent and strength of a first compact error beam at $810 \mathrm{GHz}$ following the method described e.g. by Greve et al. (1998). We find a FWHM of $240^{\prime \prime}$ corresponding roughly to the average panel size of about $0.6 \mathrm{~m}$. Only about $10-15 \%$ of the power is detected within this error beam which we attribute to panel misalignment. The power detected within a beam of $\sim 30^{\prime}$ was derived from lunar continuum scans using a lunar Rayleigh-Jeans temperature of $350 \mathrm{~K}$ for both frequency bands (Pardo et al. 2005; Mangum 1993). Moon efficiencies are $\sim 70 \%$ at both frequencies. In total, about $36 \%$ of the total power is detected in at least two error beams at both frequencies.

As the spatial source structure in each velocity channel is convolved by the antenna diagram during observations, the error beams lead to pickup of extended emission in Carina. Main beam temperatures presented here are somewhat too high. Line profiles may be slightly distorted (cf. Bensch et al. 2001). However, as the error beams are not yet completely characterized and may moreover be somewhat time variable, we ignore their influence in the following. To first order, [C I] and $\mathrm{CO}$ emission stems from the same region. Line ratios will therefore be much less affected by the error beam compared to absolute intensities.

\section{Complementary data}

\section{1. ${ }^{13} \mathrm{CO} 2-1$ data}

Brooks et al. obtained complementary maps of ${ }^{13} \mathrm{CO} 2-1$ at the Swedish-ESO Submillimetre Telescope (SEST). The telescope main beam efficiency at $220 \mathrm{GHz}$ was $50 \%$ and its HPBW 25". The northern $4^{\prime} \times 5^{\prime}$ SEST map (Brooks et al. 2003) covers the northern NANTEN2 field. The southern map (Brooks, Schneider, priv. comm.) covers the southern NANTEN2 field. We convolved these data with a Gaussian kernel to a resolution of $38^{\prime \prime}$ to allow a comparison with the NANTEN2 spectra taken along two cuts through the interface regions.

\subsection{Dust and PAHs in Carina}

Figure 1 gives an overview of a $25^{\prime} \times 25^{\prime}$ region surrounding $\eta$ Car, showing the Keyhole region in the center, and the northern and southern clouds. The OB stars of $\operatorname{Tr} 14$ and $\operatorname{Tr} 16$ emit 
far-UV photons which are partly absorbed by the dust grains of the surrounding molecular clouds. The heated dust cools in the far-infrared via dust continuum emission. Gas heating is very inefficient; only a few percent at most of the absorbed energy is transferred to the gas (Hollenbach \& Tielens 1999), which cools via far-infrared and submillimeter line emission. Since the FUV photons play a decisive role in driving the cloud chemistry, determination of the FUV field is important.

To derive a map of the estimated FUV fluxes (Fig. 1), we obtained HIRES/IRAS 60 and $100 \mu \mathrm{m}$ images at $\sim 1^{\prime}$ resolution from the IPAC data center. These images at enhanced resolution were created using a maximum correlation method (Aumann et al. 1990). The filter properties of IRAS allow us to combine these two data sets to create a map of far-infrared intensities $I_{\mathrm{FIR}}$ between $42.5 \mu \mathrm{m}$ and $122.5 \mu \mathrm{m}$ (Helou et al. 1988; Nakagawa et al. 1998). Assuming that the FUV energy absorbed by the grains is reradiated in the far-infrared, we then estimate the FUV flux $\chi(6 \mathrm{eV}<h v<13.6 \mathrm{eV})$ impinging onto the cloud surfaces from the emergent FIR intensities:

$\chi / \chi_{0}=4 \pi I_{\mathrm{FIR}} /\left(\mathrm{erg} \mathrm{s}^{-1} \mathrm{~cm}^{-2} \mathrm{sr}^{-1}\right)$

with $\chi$ in units of $\chi_{0}=2.7 \times 10^{-3} \mathrm{erg} \mathrm{s}^{-1} \mathrm{~cm}^{-2}$ (Draine 1978; Draine $\&$ Bertoldi 1996). Here, we assume that heating by photons with $h v<6 \mathrm{eV}$ contributes a factor of $\sim 2$ (Tielens \& Hollenbach 1985) and that the bolometric dust continuum intensity is a factor of $\sim 2$ larger than $I_{\text {FIR }}$ (Dale et al. 2001). In this case, the two corrections cancel out. In case the clouds do not fill the beam, the derived estimate of the FUV field will only be a lower limit. Obviously, this method fails and the FUV field cannot be derived at positions without dusty clouds.

The northern $4^{\prime} \times 4^{\prime}$ region mapped with NANTEN2 shows FUV field strengths varying between $2 \times 10^{3}$ in the north-east and $5 \times 10^{3}$ in the south-west. The FUV field in the southern region is a factor 10 weaker, varying between $4 \times 10^{2}$ in the outskirts and $8 \times 10^{2}$ near the center.

Brooks et al. (2003) estimate the FUV field at the position of peak [C II] emission in the northern cloud (Figs. 1, 3) from the spectral types of the 13 brightest stars dominating the stellar content of $\operatorname{Tr} 14$. They derive $G_{0}=1.4 \times 10^{4}$ in units of the Habing field, i.e. $\chi=8.2 \times 10^{3}$, a factor 1.6 larger than the peak flux in the northern region, as derived from HIRES. However, the value derived by Brooks et al. (2003) is a strict upper limit as it is assumed that the stars and cloud lie at the same distance to the observer and attenuation by dust can be neglected. Moreover, the FUV field derived from the stars would be lowered by smearing to the resolution of the HIRES data.

Figure 1 shows contours of the FUV field together with an $8 \mu \mathrm{m}$ MSX image. The MSX image shows the emission from very small grains and PAHs (Smith 2006a) excited by the FUV field. Evidence that the PAH emission is stimulated by FUV absorption is reflected by the good correlation between the FIR continuum and the $8 \mu \mathrm{m}$ emission.

\section{Results}

\subsection{Northern region}

\subsubsection{Maps of integrated intensities}

Figure 2 shows an overlay of integrated CO 4-3 intensities on an $8 \mu \mathrm{m}$ image taken with the Infrared Array Camera (IRAC) on the Spitzer space telescope ${ }^{1}$. The $8 \mu \mathrm{m}$ data reveal the complicated

1 These post-BCD data were retrieved from the Spitzer archive, reprojected, and merged to create Figs. 2 and 6 (cf. Smith et al. 2005).
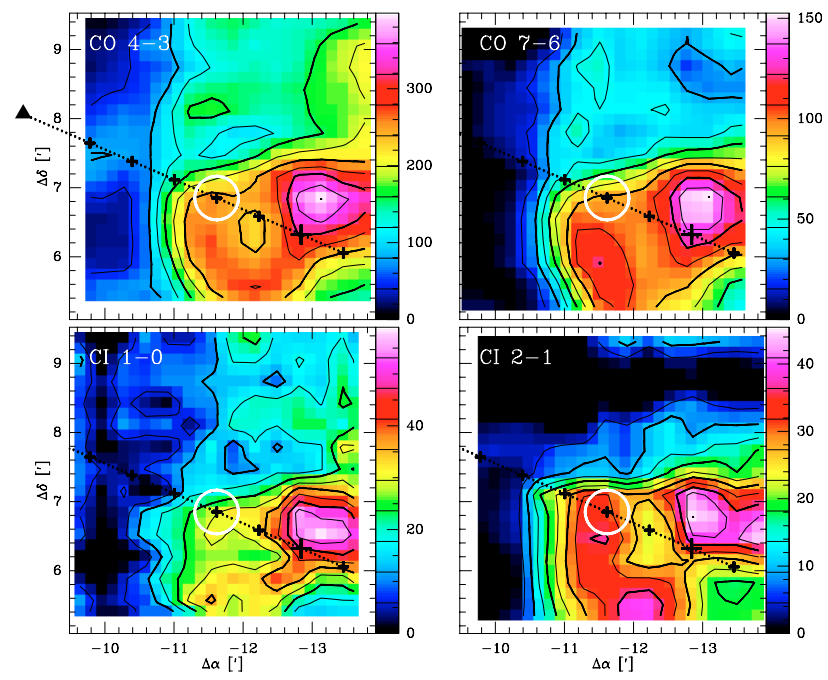

Fig. 3. Northern $4^{\prime} \times 4^{\prime}$ field: velocity-integrated maps of CO 4-3, 7-6, [CI] $1-0$, and $2-1$ at a common angular resolution of $38^{\prime \prime}(0.43 \mathrm{pc})$, integrated over the full velocity range of emission between -32 and $-5 \mathrm{~km} \mathrm{~s}^{-1}$. Contours range between 10 and $90 \%$ in steps of $10 \%$ of the peak intensities, which are $398 \mathrm{~K} \mathrm{~km} \mathrm{~s}^{-1}$ for $\mathrm{CO} 4-3,153 \mathrm{~K} \mathrm{~km} \mathrm{~s}^{-1}$ for $\mathrm{CO} 7-6,59 \mathrm{~K} \mathrm{~km} \mathrm{~s}^{-1}$ for [CI] 1-0, and $48 \mathrm{~K} \mathrm{~km} \mathrm{~s}^{-1}$ for [C I] 2-1. The center position of the compact $\mathrm{OB}$ cluster $\operatorname{Tr} 14$ is marked by a filled triangle outside the $\mathrm{CO}$ 4-3 map. A dashed line and small crosses spaced by $40^{\prime \prime}$ mark a cut through the peak of [C II] emission (Brooks et al. 2003) (large cross) and $\operatorname{Tr} 14$. The circle marks the resolution and the interface position analyzed in Table 1.

spatial structure of the region, consisting of several filaments and shell-like structures. The bright region in the south-west is the ionisation front Car I-W. Approximately $\sim 1^{\prime}$ to the east lies Car I-E (cf. Rathborne et al. 2002; Brooks et al. 2001).

Maps of integrated CO 4-3, 7-6, [C I] 1-0, and 2-1 emission (Fig. 3) show the boundary of the northern molecular cloud (orientated north-south) towards the Tr 14 cluster and its H II region. For all four tracers, the integrated intensities are co-extensive and peak in the south-west, near $-13^{\prime} /+6.5^{\prime}$, i.e. near the peak of [C II] emission studied by Brooks et al. (2003). The southeastern interface region exhibits increased CO 7-6 and [C I] 2-1 line intensities, indicating an increased amount of warm, dense gas. The morphology of the cloud resembles a ring centered on a region with less strong emission near $-12^{\prime} /+6$. $^{\prime}$. The northern half of the cloud shows weaker and more diffuse emission.

The northern region exhibits a complex velocity structure between -32 and $-5 \mathrm{~km} \mathrm{~s}^{-1}$, which can be seen in the CO 4-3 velocity channel maps (Fig. 4). Note that weak CO 4-3 emission is also detected east of the north-south interface, near $-10^{\prime} /+7.5$, at velocities between about -14.5 and $-10 \mathrm{~km} \mathrm{~s}^{-1}$. The CO 4-3 emission also corresponds well with the ${ }^{13} \mathrm{CO} 2-1$ SEST channel maps at $25^{\prime \prime}$ resolution shown in Fig. 7 of Brooks et al. (2003). The good correspondance shows that the velocity structure is largely due to individual clumps and not self-absorption of optically thick ${ }^{12} \mathrm{CO}$.

\subsubsection{Spectra along a cut through the region}

The rich kinematical structure is further revealed by the spectra along a cut, shown in Fig. 5, from the [C II] emission peak through the cloud interface, towards $\operatorname{Tr} 14$. This cut is orientated the same as the cut presented in Figs. 1 and 11 of Brooks et al. (2003). The spectra of CO 4-3, 7-6, [C I] 1-0, 2-1, and ${ }^{13}$ CO 2-1 (Brooks et al. 2003) show several velocity components 
Table 1. Physical parameters derived from the observed integrated intensities $I$ in $\mathrm{K} \mathrm{km} \mathrm{s}^{-1}$ and intensity ratios $R_{\mathrm{CO}}^{74}=I(\mathrm{CO} 7-6) / I(\mathrm{CO} 4-3)$, $R_{\mathrm{C}}^{21}=I([\mathrm{C} \mathrm{I}] 2-1) / I([\mathrm{C} \mathrm{I}] 1-0), R_{\mathrm{CCO}}^{14}=I([\mathrm{C} \mathrm{I}] 1-0) / I(\mathrm{CO} 4-3)$ from Gaussian fits to the line profiles at two interface positions. The calibration error of the integrated intensities is estimated to be $15 \%$.

\begin{tabular}{lllllllllllllrr}
\hline \hline$\Delta \alpha / \Delta \delta$ & ${ }^{13} \mathrm{CO}$ & \multicolumn{2}{c}{$\mathrm{CO}$} & \multicolumn{2}{c}{$[\mathrm{CI}]$} & $R_{\mathrm{CO}}^{74}$ & $R_{\mathrm{C}}^{21}$ & $R_{\mathrm{CCO}}^{14}$ & $T_{\text {ex }}$ & $N(\mathrm{CO})$ & $N(\mathrm{C})$ & $N\left(\mathrm{H}_{2}\right)$ & $M$ & $\mathrm{C} / \mathrm{CO}$ \\
$\left({ }^{\prime},{ }^{\prime}\right)$ & $2-1$ & $4-3$ & $7-6$ & $1-0$ & $2-1$ & & & & $\mathrm{~K}$ & & & & $M_{\odot}$ & \\
\hline$-11.7 / 6.8$ & 47 & 227 & 86 & 37 & 27 & 0.38 & 0.72 & 0.16 & 50 & $23_{18}^{30}$ & $4.9_{4.1}^{5.8}$ & $28_{21}^{35}$ & 100 & $0.21_{0.13}^{0.32}$ \\
$+0.8 /-7.2$ & 40 & 90 & 24 & 31 & 19 & 0.27 & 0.6 & 0.34 & 30 & $15_{22}^{18}$ & $3.8_{3.3}^{4.5}$ & $18_{14}^{22}$ & 63 & $0.25_{0.18}^{0.38}$ \\
\hline
\end{tabular}

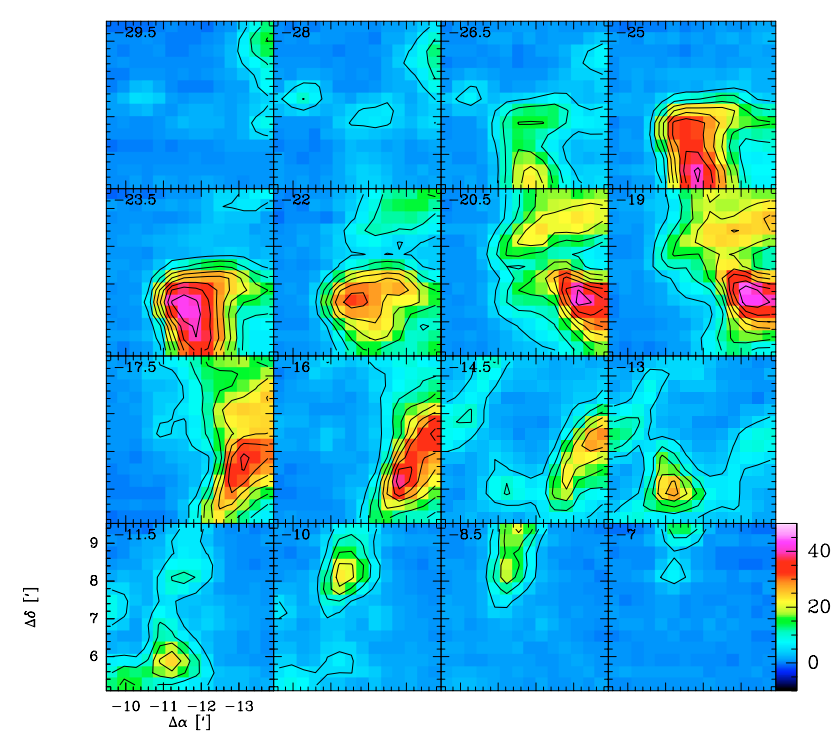

Fig. 4. Velocity structure of the northern field: $\mathrm{CO} 4-3$ velocity channels of $1.5 \mathrm{~km} \mathrm{~s}^{-1}$ width. Contours range between 5 and $45 \mathrm{~K} \mathrm{~km} \mathrm{~s}^{-1}$ in steps of $5 \mathrm{~K} \mathrm{~km} \mathrm{~s}^{-1}$.

with large velocity gradients. At the interface, all tracers show a strong component at $-24 \mathrm{~km} \mathrm{~s}^{-1}$. Further west, this component shifts to lower velocities and splits up into at least 3 components. The line centroids of the different tracers match very well. The peak temperatures are $40 \mathrm{~K}$ in $\mathrm{CO} 4-3$ and $10 \mathrm{~K}$ in [C I] $1-0$. Line intensities of $\mathrm{CO}$ and $[\mathrm{C} \mathrm{I}]$ stay constant within a factor of 2 at the positions within the cloud; they increase slightly at the interface, before dropping rapidly towards the east. A weak component at $-15 \mathrm{~km} \mathrm{~s}^{-1}$ seen in $\mathrm{CO}$ and ${ }^{13} \mathrm{CO}$ peaks to the east of the interface at $\left(-9{ }^{\prime} 8,7{ }^{\prime} 7\right)$.

To give one typical example, Table 1 shows the integrated line intensities and derived quantities of the $-24 \mathrm{~km} \mathrm{~s}^{-1}$ component at the interface position, after Gaussian fitting. $T_{\mathrm{ex}}$ is derived from the ${ }^{12} \mathrm{CO} 4-3$ peak line temperature assuming optically thick emission and a beam-filling factor of 1 . Total $\mathrm{CO}$ and $\mathrm{C}$ column densities, given in $10^{17} \mathrm{~cm}^{-2}$, were derived from ${ }^{13} \mathrm{CO} 2-1$ and [C I] $1-0$ assuming optically thin emission at $T_{\mathrm{ex}}$, LTE, and a ${ }^{12} \mathrm{CO}$ vs. ${ }^{13} \mathrm{CO}$ abundance ratio of 65 . Total $\mathrm{H}_{2}$ column densities, given in $10^{21} \mathrm{~cm}^{-2}$, and masses were derived from $N(\mathrm{CO})$ assuming an abundance ratio of $8.5 \times 10^{-5}$ (Frerking et al. 1982). $\mathrm{C} / \mathrm{CO}$ is the abundance ratio $N(\mathrm{C}) / N(\mathrm{CO})$. Errors given for the column densities were derived by varying $T_{\mathrm{ex}}$ and the integrated intensities by $\pm 15 \%$. While the ${ }^{12} \mathrm{CO} 4-3$ and $7-6$ lines show FWHMs of 5.4 and $4.7 \mathrm{~km} \mathrm{~s}^{-1}$, the [C I] 1-0 and 2-1 line widths are smaller, 3.9 and $3.1 \mathrm{~km} \mathrm{~s}^{-1} .{ }^{13} \mathrm{CO} 2-1$ shows a still narrower line width of $2.9 \mathrm{~km} \mathrm{~s}^{-1}$. We attribute this change of line widths to optical depth effects. The fitted line center velocities of all tracers agree within $0.5 \mathrm{~km} \mathrm{~s}^{-1}$. Line ratios of CO $7-6 / 4-3$ and [CI] 2-1/1-0 are 0.4 and 0.7 , which are typical values for the entire cut.

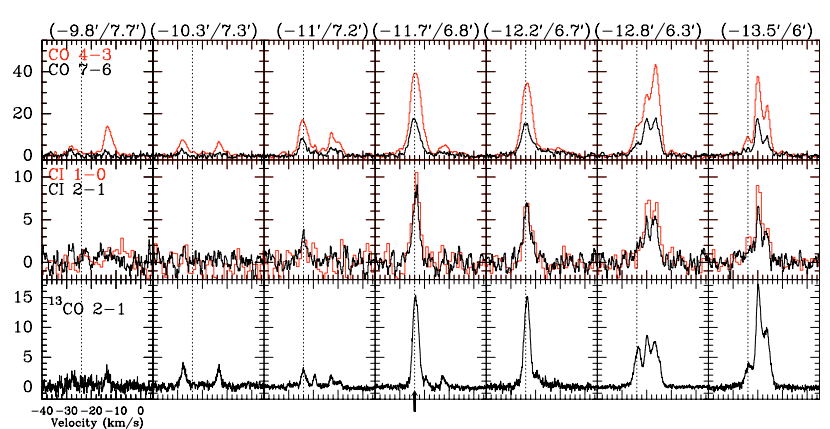

Fig. 5. Spectra along a cut through the northern field connecting $\operatorname{Tr} 14$ and the $[\mathrm{CII}]$ peak on a $40^{\prime \prime}$ grid. All data are at a common resolution of $38^{\prime \prime}$ and on the main beam scale. The velocity range is -40 to $5 \mathrm{~km} \mathrm{~s}^{-1}$. The dashed vertical lines and the arrow mark the position of the $-24 \mathrm{~km} \mathrm{~s}^{-1}$ velocity component presented in Table 1 .

The peak CO 4-3 temperature of $40 \mathrm{~K}$ translates, through the Rayleigh-Jeans correction, into a lower limit of the gas kinetic temperature of the emission zone of $50 \mathrm{~K}$. Optical depth effects or a beam-filling factor of less than 1 would imply higher gas kinetic temperatures. Brooks et al. (2003) derived a dust temperature of $50 \mathrm{~K}$ from the $60 \mathrm{vs} .100 \mu \mathrm{m}$ IRAS flux ratio at the [C II] position.

The [CI] 2-1/1-0 line ratio is a sensitive function of the [CI] excitation temperature. In the optically thin limit and assuming LTE, $T_{\mathrm{ex}}=38.3 \mathrm{~K} / \ln \left[2.11 / R_{\mathrm{CI}}^{21}\right]$. At the interface position, we observed a ratio of $0.72 \pm 0.15$, assuming a calibration error of the ratio of about $20 \%$. This translates into $T_{\mathrm{ex}}([\mathrm{CI}])$ of only $35 \mathrm{~K}\left(\begin{array}{c}43 \mathrm{~K} \\ 29 \mathrm{~K}\end{array}\right)$.

The ${ }^{13} \mathrm{CO} 2-1$ spectra (Brooks et al. 2003) peak at $15 \mathrm{~K}$. At the interface, the $[\mathrm{CI}] 1-0 /{ }^{13} \mathrm{CO} 2-1$ ratio is 0.8 .

To derive a first estimate of the total $\mathrm{CO}$ column densities at the two interface positions (Table 1), we used the ${ }^{13} \mathrm{CO} 2-1$ integrated intensities assuming optically thin emission, LTE, and a ${ }^{12} \mathrm{CO}$ vs. ${ }^{13} \mathrm{CO}$ abundance ratio of 65 . The abundance ratio is in accordance with the average local ISM ${ }^{12} \mathrm{C} /{ }^{13} \mathrm{C}$ ratio of $68 \pm 15$ recently found by Milam et al. (2005), which is valid also for Carina at about solar galacto centric distance. The inferred total optical extinction at the northern interface is $28 \pm 7 \mathrm{mag}$, using the canonical $N\left(\mathrm{H}_{2}\right) / \mathrm{A}_{\mathrm{v}}$ ratio of $9.36 \times 10^{20} \mathrm{~cm}^{-2} \mathrm{mag}^{-1}$ (Bohlin et al. 1978). The $\mathrm{C} / \mathrm{CO}$ abundance ratio is 0.21 .

\subsection{Southern region}

\subsubsection{Maps of integrated intensities}

Figure 6 shows an overlay of integrated $\mathrm{CO} 4-3$ intensities on an $8 \mu \mathrm{m}$ IRAC/Spitzer image of the southern NANTEN field. The $8 \mu \mathrm{m}$ emission traces a sharp interface running from northeast to south-west. It brightens at the cloud edge, showing several elongated filaments and knots along the interface. It also shows a bright emission knob in the north-west, which probably 


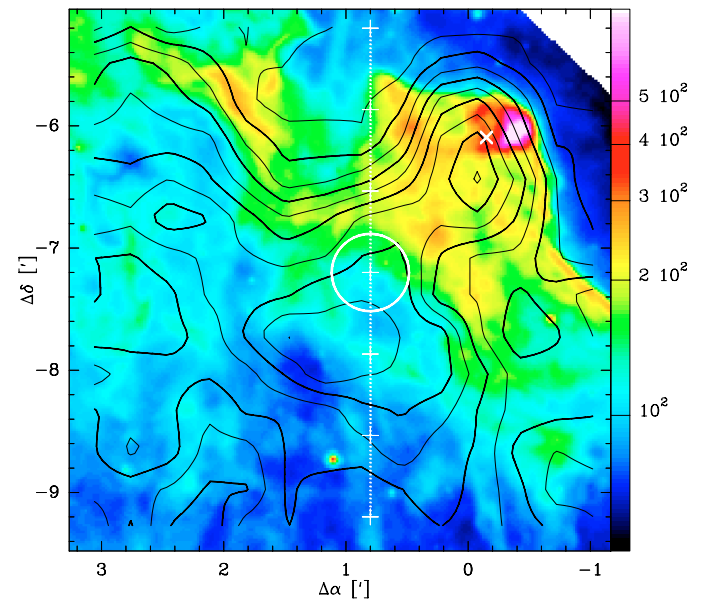

Fig. 6. Southern field: contours of integrated $\mathrm{CO} 4-3$ intensities (cf. Fig. 7) overlayed on $8 \mu \mathrm{m} \mathrm{IRAC/Spitzer} \mathrm{emission} \mathrm{in} \mathrm{units} \mathrm{of} \mathrm{MJy/sr.}$

corresponds to the IRAS point source 10430-5931. The $8 \mu \mathrm{m}$, emission here is weaker than in the northern field by about a factor 5. The IRAC emission drops rapidly towards the bulk of the cloud in the south-east, similar to PAH emission traced by the $3.21 \mu \mathrm{m}$ image of Rathborne et al. (2002).

In contrast to the mid-infrared emission, $\mathrm{CO} 4-3$ and [C I] integrated intensities (Fig. 7) peak south of the interface deep inside the cloud near $\left(1^{\prime},-8^{\prime}\right)$. The $\mathrm{CO} 7-6$ line peaks near IRAS 10430-5931, indicating elevated temperatures and densities. In contrast, the IRAS source is not prominent in [C I].

\subsubsection{Spectra along a cut through the region}

Spectra taken along a north-south cut through the southern field show one component near $-27 \mathrm{~km} \mathrm{~s}^{-1}$ with little variation in velocity (Fig. 8). Near the interface, ${ }^{12} \mathrm{CO}$ line profiles are flat-topped indicating high optical depths and self-absorption. Table 1 lists the results of Gaussian fits to the spectra at the interface position $(0,8,-7 ! 3)$. The fitted $F W H M$ s are $4.3 \mathrm{~km} \mathrm{~s}^{-1}$ (CO 4-3), $3.9 \mathrm{~km} \mathrm{~s}^{-1}$ (CO 7-6), $3.4 \mathrm{~km} \mathrm{~s}^{-1}$ ( ${ }^{13} \mathrm{CO} 2-1$ ), $2.1 \mathrm{~km} \mathrm{~s}^{-1}$ ([C I $\left.] 1-0\right), 2.4 \mathrm{~km} \mathrm{~s}^{-1}$ ([C I $\left.] 2-1\right) .{ }^{12} \mathrm{CO}$ line widths are a factor $\sim 2$ broader than the $[\mathrm{CI}]$ line widths which probably reflects changes of the optical depths.

Compared to the northern field, the ${ }^{12} \mathrm{CO}$ and ${ }^{13} \mathrm{CO}$ lines are weaker while the [C I] 1-0 line is stronger. The $\mathrm{CO} 4-3$ peaks at only $\sim 20 \mathrm{~K}$. The line ratios of CO 7-6/4-3 and [C I] 2-1/1-0 at the interface position are $0.3 \pm 20 \%$ and $0.6 \pm 20 \%$ respectively. These ratios are slightly lower than in the northern field, suggesting lower temperatures and densities. However, optical depth effects and self-absorption may also play a role. The [C I ] 1-0/ $\mathrm{CO} 4-3$ ratio is 0.34 at the interface, a factor 2 higher than in the northern interface. The large-scale survey of the entire Carina region by Zhang et al. (2001) showed variations between 0.14 and 0.45 , similar to the ratios we find at angular resolutions which are a factor $\sim 5$ higher. Gas kinetic temperatures must be at least $30 \mathrm{~K}$ to explain the ${ }^{12} \mathrm{CO} 4-3$ main beam temperatures. In the southern interface, this is consistent with $T_{\mathrm{ex}}([\mathrm{CI}])=30.5 \mathrm{~K}$ $(26 \mathrm{~K})$ derived from the $[\mathrm{CI}]$ line ratio.

The LTE analysis at the southern position indicates a C/CO abundance ratio of 0.26 , slightly higher than the abundance ratio in the north. Taking into account the calibration errors, the $\mathrm{C} / \mathrm{CO}$ abundance ratios lie between $\sim 0.1$ and $\sim 0.4$ at both positions (Table 1). Similar abundance ratios have been observed in other
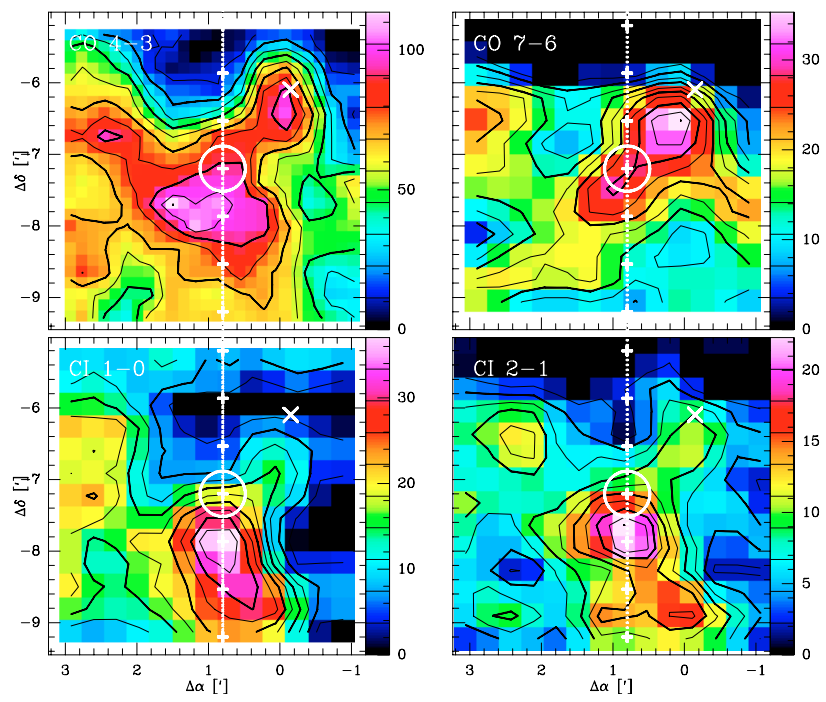

Fig. 7. Southern $4^{\prime} \times 4^{\prime}$ field: velocity-integrated maps of CO 4-3, 7-6, [C I] $1-0$, and $2-1$ at a common angular resolution of $38^{\prime \prime}$, integrated over the full velocity range of emission between -20 and $-32 \mathrm{~km} \mathrm{~s}^{-1}$. Contours range between 10 and $90 \%$ in steps of $10 \%$ of the peak intensities which are $114 \mathrm{~K} \mathrm{~km} \mathrm{~s}^{-1}$ for $\mathrm{CO} 4-3,35 \mathrm{~K} \mathrm{~km} \mathrm{~s}^{-1}$ for CO 7-6, $37 \mathrm{~K} \mathrm{~km} \mathrm{~s}^{-1}$ for [CI] 1-0, and $22 \mathrm{~K} \mathrm{~km} \mathrm{~s}^{-1}$ for [CI] 2-1. A cross $\times$ marks the position of IRAS $10430-5931$ at $10^{\mathrm{h}} 45^{\mathrm{m}} \cdot 02^{\mathrm{s}} .4-59.47{ }^{\prime} \cdot 10^{\prime} 0$ (J2000). Small crosses + mark positions along a cut through the interface region. The circle marks the resolution and the position analyzed in Table 1.

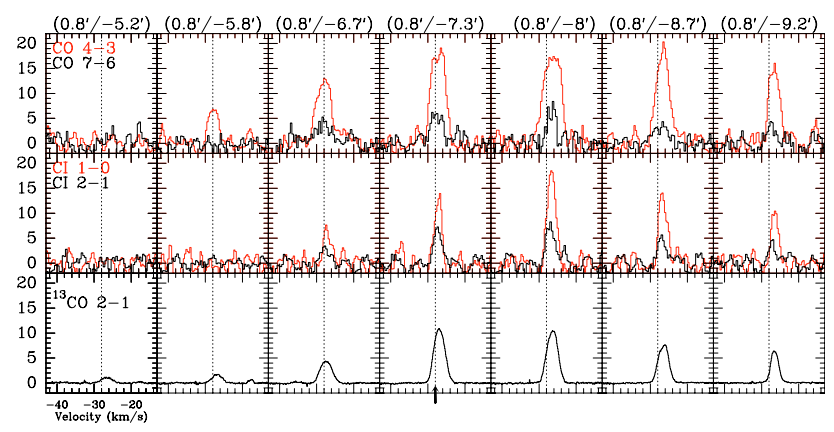

Fig. 8. Spectra along a north-south orientated cut through the southern field at $\Delta \alpha=0.8$ on a $40^{\prime \prime}$ grid. All data are at a common resolution of $38^{\prime \prime}$. The velocity range is -43 to $-13 \mathrm{~km} \mathrm{~s}^{-1}$. Vertical lines mark a velocity of $-28 \mathrm{~km} \mathrm{~s}^{-1}$. The arrow marks the position analyzed in Table 1.

Galactic star forming regions like Cepheus B or NGC 7023 (see references in Mookerjea et al. 2006).

\section{Clumpy interface regions}

Figure 9 shows the cooling intensities ${ }^{2}$ observed at the two interface positions (Table 1) along the cuts presented in the previous section. At both positions, the 7-6 intensity exceeds the 4-3 intensity. As this transition has a critical density of $1.4 \times 10^{6} \mathrm{~cm}^{-3}$ and an upper-level energy corresponding to $157 \mathrm{~K}$, temperatures and densities are expected to be similarly high.

In the following, we compare the observed emission with the predictions of a clumpy PDR model (Cubick 2005; Cubick et al. 2007). We assume that the emission stems from an ensemble of spherically symmetric clumps within the beam. The

\footnotetext{
2 We used $\int I_{\nu} \mathrm{d} v\left[\operatorname{erg~s}^{-1} \mathrm{~cm}^{-2} \mathrm{sr}^{-1}\right]=\frac{2 \mathrm{k} \nu^{3}}{c^{3}} \int T \mathrm{~d} v\left[\mathrm{~K} \mathrm{~km} \mathrm{~s}^{-1}\right]$.
} 
Table 2. Parameters of the clump ensembles at the two positions which fit best the observed intensities. Columns (2) to (6) list the input parameters from which the values given in Cols. (7) to (11) are derived: $\chi$ is the FUV field in Draine units, $\langle n\rangle_{\text {ens }}$ is the average density of the clump ensemble, $M_{\mathrm{cl}}^{\min }, M_{\mathrm{cl}}^{\max }, M_{\mathrm{tot}}$ are the minimum and maximum clump mass, and the total ensemble mass, respectively. $n_{0, \min }$ and $n_{0, \max }$ are the smallest and largest clump surface densities, $R_{\min }$ and $R_{\max }$ are the smallest and largest clump radii, and $\phi_{\mathrm{A}}$ is the beam-filling factor of the clump ensemble.

\begin{tabular}{|c|c|c|c|c|c|c|c|c|c|c|}
\hline $\begin{array}{l}\Delta \alpha / \Delta \delta \\
\left({ }^{\prime},{ }^{\prime}\right) \\
(1)\end{array}$ & $\begin{array}{l}x \\
(2)\end{array}$ & $\begin{array}{l}\langle n\rangle_{\text {ens }} \\
\mathrm{cm}^{-3} \\
(3) \\
\end{array}$ & $\begin{array}{l}M_{\mathrm{cl}}^{\min } \\
M_{\odot} \\
(4) \\
\end{array}$ & $\begin{array}{l}M_{\mathrm{cl}}^{\max } \\
M_{\odot} \\
(5) \\
\end{array}$ & $\begin{array}{l}M_{\text {tot }} \\
M_{\odot} \\
(6) \\
\end{array}$ & $\begin{array}{l}n_{0, \min } \\
\mathrm{cm}^{-3} \\
(7)\end{array}$ & $\begin{array}{l}n_{0, \max } \\
\mathrm{cm}^{-3} \\
(8)\end{array}$ & $\begin{array}{l}R_{\min } \\
\mathrm{pc} \\
(9) \\
\end{array}$ & $\begin{array}{r}R_{\max } \\
\mathrm{pc} \\
(10) \\
\end{array}$ & $\begin{array}{r}\phi_{\mathrm{A}} \\
(11) \\
\end{array}$ \\
\hline$-11.7 / 6.8$ & $10^{3.5}$ & $2 \times 10^{5}$ & $10^{-2}$ & $10^{2}$ & 400 & $5.3 \times 10^{4}$ & $8.8 \times 10^{5}$ & $3.7 \times 10^{-3}$ & $2.1 \times 10^{-1}$ & 3.9 \\
\hline$+0.8 /-7.2$ & $10^{2.5}$ & $2 \times 10^{5}$ & $10^{-2}$ & $10^{2}$ & 220 & $5.3 \times 10^{4}$ & $8.8 \times 10^{5}$ & $3.7 \times 10^{-3}$ & 0.2 & 2.1 \\
\hline
\end{tabular}

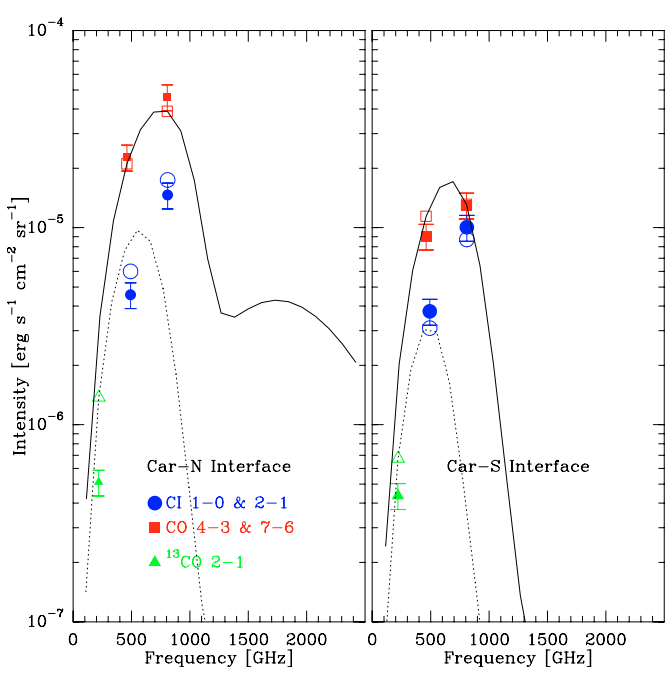

Fig. 9. Integrated intensities at the two interface positions in CarinaNorth and South (Table 1). Filled symbols show the observed ${ }^{13} \mathrm{CO} 2-1$, [C I] 1-0, 2-1, ${ }^{12} \mathrm{CO} 4-3$, and 7-6 intensities. Error bars denote the $15 \%$ calibration error. Model results of the best fitting clumpy PDR model (Table 2$)$ are shown by solid $\left({ }^{12} \mathrm{CO}\right)$ and dotted lines $\left({ }^{13} \mathrm{CO}\right)$, and open symbols.

clumps follow the canonical clump mass and mass size distributions: $\mathrm{d} N / \mathrm{d} M \propto M^{-\alpha}$ and $M \propto R^{\gamma}$. For the clump mass spectrum derived from molecular line observations, $\alpha \sim 1.8$ in a large number of Galactic clouds (e.g. Kramer et al. 1998; Simon et al. 2001). For the power law describing the mass-size distribution, $\gamma \sim 2.3$ in several Galactic clouds (e.g. Heithausen et al. 1998). As a result, the clump density distribution is $n \propto M^{1-3 / \gamma}$, i.e. the density increases with decreasing clump mass. For the nearby clouds L 1457 and the Polaris Flare presented in the Kramer and Heithausen papers, the canonical clump mass and mass size distributions continue down to masses of less than $10^{-3} M_{\odot}$ and radii of less than $10^{-2} \mathrm{pc}$.

The emission from a clump of given mass and density is calculated by the KOSMA- $\tau$ model (Röllig et al. 2006; Störzer et al. 1996). The radial density structure $n(r)$ of a model clump with radius $R_{0}$ is given by $n(r) \propto n_{0}\left(r / R_{0}\right)^{-1.5}$ for $0.2 \leq r / R_{0} \leq 1$ and $n(r)=11.18 n_{0}=$ const. for $r / R_{0} \leq 0.2$. The clump averaged density $\langle n\rangle$ is about twice the density at the clump surface, called henceforth surface density $n_{0}:\langle n\rangle \sim 1.91 n_{0}$. PDR models of individual clumps were calculated on a grid of FUV fields $\log (\chi)=0,0.5$, to 6 , clump surface densities $\log \left(n_{0} / \mathrm{cm}^{-3}\right)=$ $2,2.5$, to 7 , and clump masses $\log \left(M_{\mathrm{cl}} / M_{\odot}\right)=-3,-2.5$, to 2 . The parameters controlling the ensemble model are the FUV field, the average ensemble density, the total ensemble mass, and the lower and upper clump mass limits. Here, we assume that all clumps are heated by a constant FUV field, as derived from the HIRES observations presented above. We chose a lower
Table 3. Ratios of observed integrated intensities over integrated intensities predicted by the clumpy PDR model. The input parameters used are given in Table 2; deviations from this setting are listed in Col. (7).

\begin{tabular}{lllllll}
\hline \hline$\Delta \alpha / \Delta \delta$ & ${ }^{13} \mathrm{CO}$ & \multicolumn{3}{c}{$\mathrm{CO}$} & \multicolumn{1}{c}{$\mathrm{I}]$} \\
$\left({ }^{\prime},\right)$ & $2-1$ & $4-3$ & $7-6$ & $1-0$ & $2-1$ & \\
$(1)$ & $(2)$ & $(3)$ & $(4)$ & $(5)$ & $(6)$ & $(7)$ \\
\hline$-11.7 / 6.8$ & 0.4 & 1.1 & 1.2 & 0.8 & 0.8 & \\
$-11.7 / 6.8$ & 0.3 & 0.9 & 0.8 & 0.7 & 0.8 & $M_{\min }=10^{-3} M_{\odot}$ \\
$-11.7 / 6.8$ & 0.4 & 1.3 & 1.7 & 0.8 & 1 & $M_{\min }=10^{-1} M_{\odot}$ \\
$-11.7 / 6.8$ & 0.5 & 1.7 & 1.5 & 1.4 & 1.5 & $M_{\max }=10^{3} M_{\odot}$ \\
$-11.7 / 6.8$ & 0.3 & 0.8 & 1 & 0.5 & 0.5 & $M_{\max }=10^{1} M_{\odot}$ \\
\hline$+0.8 /-7.2$ & 0.6 & 0.8 & 1 & 1.2 & 1.2 & \\
\hline
\end{tabular}

mass cutoff of the ensemble of $10^{-2} M_{\odot}$ and an upper cutoff of $100 M_{\odot}$. Below, we show that the exact choice of these limits does however not have a strong impact on the resulting model intensities (cf. Cubick et al. 2007). To fit the observed intensity ratios [C I ] 2-1/1-0 and CO 7-6/4-3, the only free parameter is then the average density of the clump ensemble $\langle n\rangle_{\mathrm{ens}}$.

We adapted the total ensemble mass to fit the absolute intensities. The best fitting models and their parameters are shown in Fig. 9 and in Table 2. Table 3 lists the ratios of observed over modelled intensities for all 5 observed transitions. For a perfectly fitting model, they would all equal $1 \pm 15 \%$, reflecting the estimated calibration error of the observed data.

Northern interface. An average ensemble density of $2 \times$ $10^{5} \mathrm{~cm}^{-3}$ allows us to reproduce the observed ${ }^{12} \mathrm{CO} 7-6 / 4-3$ and [C I ] 2-1/1-0 line ratios very well at the northern interface position. Lower densities do not reproduce the high CO 7-6/4-3 line ratios, but rather lead to a peak of the cooling curve at $J \leq 5$. The absolute intensities of the ${ }^{12} \mathrm{CO}$ and ${ }^{12} \mathrm{C}$ lines can be reproduced to within $20 \%$ for an ensemble mass of $400 M_{\odot}$. The strongest deviation occurs for ${ }^{13} \mathrm{CO}$; the observed ${ }^{13} \mathrm{CO} 2-1$ line intensity is only $40 \%$ of the modelled intensity.

The geometrical beam-filling factor of the clump ensemble is defined as the ratio of the sum of all clump areas over the beam area, i.e. $\phi_{\mathrm{A}}=\Omega_{\mathrm{ens}} / \Omega_{\mathrm{beam}}$, with $\Omega_{\mathrm{ens}}=\sum \pi R_{\mathrm{cl}}^{2} / d^{2}$ at distance $d$. The beam-filling factor is 3.9 at the northern interface position (Table 2). We assume that clumps don't overlap, i.e. that their emission escapes without being absorbed by foreground clumps, and there is no interclump medium. Clumps have a constant velocity $F W H M$ of $\Delta v=1.7 \mathrm{~km} \mathrm{~s}^{-1}$. The clumpy ensemble models developed by Cubick et al. (2007) do not yet predict line profiles. Table 2 gives the densities and radii of the smallest and largest clumps of the ensemble. The mass of the smallest clump is set to $10^{-2} M_{\odot}$. The virial mass of the smallest clump, $M_{\text {vir }} \propto R \Delta v^{2}$ is a factor 400 larger. In other words, the velocity width of these clumps are far too large for pure gravitational virialization. The external pressure which would be needed to 
confine these clumps, $10^{8} \mathrm{~K} \mathrm{~cm}^{-3}$, is far too large to be maintained by an interclump medium. The large ratio indicates instead that these clumps, if they do exist, are transient features of the turbulent gas and evaporating on very short time scales: $t_{\text {evap }} \approx R / \sigma_{\mathrm{v}}=R / F W H M \sqrt{8 \ln 2} \approx 5000$ yrs.

The modelled intensities depend only weakly on the lower and upper mass limits of the ensemble as shown in Table 3. Varying one of the mass limits by one order of magnitude while keeping all other 4 input parameters listed in Table 2 unchanged, the absolute intensities of the 5 transitions vary by less than a factor of $\sim 2$ relative to the best fitting solution. This is discussed in more detail in Cubick et al. (2007).

The run of ${ }^{12} \mathrm{CO}$ intensities with rotational number $J$, i.e. the $\mathrm{CO}$ cooling curve, predicted by the model (Fig. 9) shows a maximum at $J=7$ and a steep drop at higher rotational numbers up to $J=11$ by more than a magnitude. A secondary peak of the cooling curve is seen at $J=15$; it is almost a factor 10 weaker than the first maximum. The second peak shows up only for FUV fields above $\sim 10^{3}$ and it stems predominantly from the surface regions of the small, dense clumps. These clumps are large in number, have a large total surface area, which is heated to high temperatures, sufficient to excite these high- $J$ lines. The abundance of $\mathrm{CO}$ is increased in the hot gas of the $\mathrm{H} \mathrm{I} / \mathrm{H}_{2}$ surface layer, near the $\mathrm{OH}$ density peak, due to secondary production paths which become important at high temperatures (Sternberg \& Dalgarno 1995):

$$
\begin{aligned}
\mathrm{OH}+\mathrm{C}^{+} & \rightarrow \mathrm{CO}+\mathrm{H}^{+} \quad(\mathrm{R} 13) \\
\mathrm{OH}+\mathrm{C}^{+} & \rightarrow \mathrm{CO}^{+}+\mathrm{H} \rightarrow \mathrm{CO}+\mathrm{H}^{+} \\
\mathrm{CO}^{+}+\mathrm{H}_{2} & \rightarrow \mathrm{HCO}^{+}+\mathrm{H} \quad(\mathrm{R} 127) \\
\mathrm{HCO}^{+}+e & \rightarrow \mathrm{CO}+\mathrm{H} \quad(\mathrm{R} 129) .
\end{aligned}
$$

We suspect that these reactions lead to the secondary peak of the cooling curve shown in Fig. 9.

Southern interface. At the southern interface position, the FUV field is only $10^{2.5} \chi_{0}$. An average ensemble density of $2 \times 10^{5} \mathrm{~cm}^{-3}$ reproduces the observed ${ }^{12} \mathrm{CO} 7-6 / 4-3$ and [C I] 2-1/1-0 line ratios. Within $40 \%$, the clumpy model is also consistent with the absolute intensities of all 5 tracers for a total ensemble mass of $220 M_{\odot}$ (Fig. 9, Tables 3,2). Due to the weaker FUV field compared to the northern position, the $\mathrm{CO}$ cooling curve peaks at slightly lower frequencies and a second peak is not visible. Similar to the northern position, the strongest deviation occurs for the ${ }^{13} \mathrm{CO} 2-1$ lines.

\section{Summary}

We have mapped the emission of atomic carbon and $\mathrm{CO}$ in two fields of the GMCs surrounding $\eta$ Car. Combining CO 4-3 and [C I] 1-0 data with $\mathrm{CO} 7-6$ and [CI] 2-1 data allows us to study the excitation conditions of both gas tracers. The northern $4^{\prime} \times 4^{\prime}$ field lies adjacent to the compact OB cluster Tr 14 and is associated with the H II regions Car I-E and Car I-W. The spectral lines show a rich kinematical structure, with several velocity components along the lines of sight spread between -10 and $-30 \mathrm{~km} \mathrm{~s}^{-1}$. The southern $4^{\prime} \times 4^{\prime}$ field lies on a molecular ridge south of $\eta$ Car. Here, spectral lines show only one rather narrow Gaussian shaped velocity component. The FUV field derived from HIRES/IRAS far-infrared data is $\chi \sim 10^{2.5}$, about a factor 10 weaker than in the northern field. IRAC/Spitzer maps at $8 \mu \mathrm{m}$ show the detailed structure of the FUV illuminated dust. The edge of the southern region is clearly delineated by the $8 \mu \mathrm{m}$ emission, while the northern region shows a more complex spatial structure.

In both regions, $\mathrm{CO}$ and $[\mathrm{C} \mathrm{I}]$ emission is co-extensive. Both species appear to trace the bulk of molecular gas, rather than the interface regions. This coincidence has been found in many of the previous large-scale studies of Galactic clouds conducted with the Mt. Fuji, KOSMA, and other telescopes. Papadopoulos et al. (2004) stress the importance of cosmic rays in raising the $\mathrm{C} / \mathrm{CO}$ abundance ratio and argue that dynamic and nonequilibrium chemistry processes explain the ubiquity of [CI] within molecular clouds as well as at PDR interfaces. Here, we argue that steady state, clumpy PDR models provide an alternative explanation.

The northern and southern Carina regions both show a slight brightening of the $\mathrm{CO} 7-6$ and $[\mathrm{C}$ I] 2-1 transitions relative to the 4-3 and 1-0 transitions near the interfaces. In the south-east part of the northern region, the upper transitions brighten towards the interface (Fig. 3). These transitions also show increased intensities near the embedded IRAS point source in the southern region (Fig. 7).

We selected two cuts from the cloud cores through the interface regions, roughly pointing towards the illuminating sources. The southern cut lies to the east of IRAS 10430-5931, avoiding its complicated structure and additional heating sources. The northern cut runs from the peak of [C II] emission (Brooks et al. 2003) through the H II regions Car I-W and I-E across the edgeon interface towards the center of the $\operatorname{Tr} 14$ cluster.

The peak ${ }^{12} \mathrm{CO}$ line temperatures along the northern cut indicate kinetic gas temperatures of at least $50 \mathrm{~K}$, while a lower limit of $30 \mathrm{~K}$ is found along the southern cut.

We selected two positions at the northern and southern interfaces for a detailed analysis. The ${ }^{12} \mathrm{CO} 7-6 / 4-3$ and $[\mathrm{C} \mathrm{I}] 2-$ $1 / 1-0$ intensity ratios at these positions are $0.3-0.4$ and $0.6-0.7$, respectively. We use PDR models to interpret the observed line intensities. Our models assume that all observed emission stems from an ensemble of spherically symmetric PDR clumps and there is no emission from an interclump medium or a diffuse halo surrounding the clouds. PDR clumps are modelled using the stationary KOSMA- $\tau$ code (Röllig et al. 2006). The clump distributions follow the canonical mass and radius distributions found for molecular clouds. At the two positions analyzed in detail, we find that clumpy PDR models are consistent with the observed absolute intensities of the ${ }^{12} \mathrm{CO}$ and [CI] lines to within $20 \%$, i.e. at about the calibration accuracy.

Since the line ratios observed at the two interface positions are fairly typical for the entire observed regions, we conclude that stationary, clumpy PDR models can simultaneously reproduce the observed $[\mathrm{CI}]$ and $\mathrm{CO}$ emission of the lower transitions and the upper $\mathrm{CO} 7-6$ and $[\mathrm{C} \mathrm{I}] 2-1$ transitions.

However, the observed ${ }^{13} \mathrm{CO} 2-1$ intensities are only about half of the modelled intensities at the northern and southern positions, respectively. Interestingly, Pineda \& Bensch (2007) report a similar finding. They analyzed ${ }^{12} \mathrm{CO}$ and $[\mathrm{CI}]$ emission observed in the dark cloud globule B 68. This emission can be reproduced using a single KOSMA- $\tau$ spherically symmetric PDR model, while the observed ${ }^{13} \mathrm{CO}$ intensities are a factor $\sim 2$ too low.

To study the gas under a broad range of conditions, we plan to extend the present $[\mathrm{CI}]$ and $\mathrm{CO}$ maps to fully cover the GMCs surrounding $\eta$ Car using the SMART $492 / 810 \mathrm{GHz}$ multipixel array receiver at NANTEN2 (Graf et al. 2003). Future observations with APEX and Herschel will tell in how far the emission of other species tracing the detailed photo-chemical network is also consistent with stationary, clumpy PDR models. 
Acknowledgements. We thank Kate Brooks and Nicola Schneider for making their partly unpublished SEST ${ }^{13} \mathrm{CO} 2-1$ data available to us. We also would like to thank an anonymous referee for insightful comments which helped to improve on our arguments. We made use of the NASA/IPAC/IRAS/HiRES data reduction facilities. Data reduction of the spectral line data was done with the gildas software package supported at IRAM (see http://www.iram.fr/IRAMFR/GILDAS). This research has made use of NASA's Astrophysics Data System Abstract Service.

The NANTEN2 project (southern submillimeter observatory consisting of a 4-meter telescope) is based on a mutual agreement between Nagoya University and the University of Chile and includes member universities from six countries, Australia, Republic of Chile, Germany, Japan, Republic of Korea, and Swiss Confederation. We acknowledge that this project could be realized by financial contributions of many Japanese donators and companies.

This work is financially supported in part by a Grant-in-Aid for Scientific Research from the Ministry of Education, Culture, Sports, Science and Technology of Japan (No. 15071203) and from JSPS (Nos. 14102003 and 18684003), and by the JSPS core-to-core program (No. 17004). This work is also financially supported in part by the grant SFB 494 of the Deutsche Forschungsgemeinschaft, the Ministerium für Innovation, Wissenschaft, Forschung und Technologie des Landes Nordrhein-Westfalen and through special grants of the Universität zu Köln and Universität Bonn. L.B. and J.M. acknowledge support from the Chilean Center for Astrophysics FONDAP 15010003 .

\section{References}

Aumann, H., Fowler, J., \& Melnyk, M. 1990, AJ, 99, 1674

Bayet, E., Gerin, M., Phillips, T. G., \& Contursi, A. 2006, A\&A, 460, 467

Bensch, F. 2006, A\&A, 448, 1043

Bensch, F., Stutzki, J., \& Heithausen, A. 2001, A\&A, 365, 285

Bensch, F., Leuenhagen, U., Stutzki, J., \& Schieder, R. 2003, A\&A, 591, 1013

Bohlin, R. C., Savage, B. D., \& Drake, J. F. 1978, ApJ, 224, 132

Boissé, P. 1990, A\&A, 228, 483

Brooks, K. J., Storey, J. W. V., \& Whiteoak, J. B. 2001, MNRAS, 327, 46

Brooks, K., Cox, P., Schneider, N., et al. 2003, A\&A, 412, 751

Cox, P. 1995, in Rev. Mex. Astron. Astrofis. Conf. Ser., ed. V. Niemela, N. Morrell, \& A. Feinstein, 105

Cubick, M. 2005, Modelling the FIR emission of the Milky Way, Diploma thesis, Universität zu Köln

Cubick, M., Stutzki, J., Ossenkopf, V., Kramer, C., \& Röllig, M. 2007, A\&A, in prep.

Dale, D. A., Helou, G., Contursi, A., Silbermann, N. A., \& Kolhatkar, S. 2001, ApJ, 549, 215

Draine, B. T. 1978, ApJS, 36, 595

Draine, B., \& Bertoldi, F. 1996, ApJ, 468, 269

Fixsen, D. J., Bennett, C. L., \& Mather, J. C. 1999, ApJ, 526, 207

Frerking, M. A., Langer, W. D., \& Wilson, R. W. 1982, ApJ, 262, 590

Grabelsky, D. A., Cohen, R. S., Bronfman, L., \& Thaddeus, P. 1988, ApJ, 331, 181
Graf, U., Heyminck, S., Michael, E., et al. 2003, in Millimeter and Submillimeter Detectors for Astronomy, ed. T. G. Phillips, \& J. Zmuidzinas, Proc. SPIE, 4855,322

Greve, A., Kramer, C., \& Wild, W. 1998, A\&AS, 133, 271

Griffin, M. J., Ade, P., Orton, G., et al. 1986, Icarus, 65, 244

Hegmann, M., Kegel, W. H., \& Sedlmayr, E. 2007, A\&A, 469, 223

Heithausen, A., Bensch, F., Stutzki, J., Falgarone, E., \& Panis, J. 1998, A\&A, 331, 65

Helou, G., Khan, I., Malek, L., \& Boehmer, L. 1988, ApJ, 68, 151

Hollenbach, D. J., \& Tielens, A. G. G. M. 1999, Rev. Mod. Phys., 71, 173

Jakob, H., Kramer, C., Simon, R., et al. 2007, A\&A, 461, 999

Juvela, M., Padoan, P., \& Nordlund, Å. 2001, ApJ, 563, 853

Kramer, C., Stutzki, J., Röhrig, R., \& Corneliussen, U. 1998, A\&A, 329, 249

Kramer, C., Mookerjea, B., Bayet, E., et al. 2005, A\&A, 441, 961

Mangum, J. G. 1993, PASP, 105, 117

Meixner, M., \& Tielens, A. G. G. M. 1993, ApJ, 405, 216

Milam, S. N., Savage, C., Brewster, M. A., Ziurys, L. M., \& Wyckoff, S. 2005, ApJ, 634, 1126

Mizutani, M., Onaka, T., \& Shibai, H. 2002, A\&A, 382, 610

Mizutani, M., Onaka, T., \& Shibai, H. 2004, A\&A, 423, 579

Mookerjea, B., Kramer, C., Röllig, M., \& Masur, M. 2006, A\&A, 456, 235

Nakagawa, T., Yui, Y. Y., Doi, Y., et al. 1998, ApJS, 115, 259

Oberst, T. E., Parshley, S. C., Stacey, G. J., et al. 2006, ApJ, 652, L125

Oka, T., Iwata, M., Maezawa, H., et al. 2004, ApJ, 602, 803

Papadopoulos, P. P., Thi, W.-F., \& Viti, S. 2004, MNRAS, 351, 147

Pardo, J., Cernicharo, J., \& Serabyn, E. 2001, IEEE Trans. on Antennas and Propagation, 49, 1683

Pardo, J. R., Serabyn, E., \& Wiedner, M. C. 2005, Icarus, 178, 19

Pineda, J. L., \& Bensch, F. 2007, A\&A, 470, 615

Rathborne, J. M., Burton, M. G., Brooks, K. J., et al. 2002, MNRAS, 331, 85

Röllig, M., Ossenkopf, V., Jeyakumar, S., Stutzki, J., \& Sternberg, A. 2006, A\&A, 451, 917

Röllig, M., Abel, N. P., Bell, T., et al. 2007, A\&A, 467, 187

Sakai, T., Oka, T., \& Yamamoto, S. 2006, ApJ, 649, 268

Schulz, A., Henkel, C., Muders, D., et al. 2007, A\&A, 466, 467

Simon, R., Jackson, J. M., Clemens, D. P., Bania, T. M., \& Heyer, M. H. 2001, ApJ, 551, 747

Simon, R., Graf, U., Kramer, C., Stutzki, J., \& Onishi, T. 2007, in NANTEN technical report, 13.2.2007, 1

Smith, N. 2006a, MNRAS, 367, 763

Smith, N. 2006b, ApJ, 644, 1151

Smith, N., \& Brooks, K. J. 2007, MNRAS, 379, 1279

Smith, N., Churchwell, E. B., Whitney, B., et al. 2005, in BAAS, 439

Spaans, M. 1996, A\&A, 307, 271

Spaans, M., \& van Dishoeck, E. F. 1997, A\&A, 323, 953

Sternberg, A., \& Dalgarno, A. 1995, ApJ, 99, 565

Störzer, H., Stutzki, J., \& Sternberg, A. 1996, A\&A, 310, 592

Störzer, H., Stutzki, J., \& Sternberg, A. 1997, A\&A, 323, 13

Tatematsu, K., Jaffe, D., Plume, R., \& Evans, N. 1999, ApJ, 526, 295

Tielens, A., \& Hollenbach, D. 1985, ApJ, 291, 722

Weiss, A., Henkel, C., Downes, D., \& Walter, F. 2003, A\&A, 409, 41

Yonekura, Y., Asayama, S., Kimura, K., et al. 2005, ApJ, 634, 476

Zhang, X., Lee, Y., Bolatto, A., \& Stark, A. A. 2001, ApJ, 553, 274 